\title{
Associations of chronotype with clock genes polymorphisms
}

\author{
Mirkka Maukonen $^{1}$, Aki Havulinna ${ }^{1,2}$, Timo Partonen $^{1}$, Noora Kanerva ${ }^{3}$ and Satu Männistö ${ }^{1}$ \\ ${ }^{1}$ National Institute for Health and Welfare, Helsinki, Finland, \\ ${ }^{2}$ Institute for Molecular Medicine Finland (FIMM/HiLIFE), Helsinki, Finland and \\ ${ }^{3}$ Nightingale, Helsinki, Finland
}

\begin{abstract}
Introduction: Individuals with a later timing of circadian rhythms (evening types) tend to have unhealthier behaviors compared to those with an earlier timing (morning types). Previous studies have shown that evening types have unhealthier diets and later eating times than morning types. Furthermore, evening types bear higher odds for type 2 diabetes and weight gain (women). Circadian rhythms are controlled by clock genes which have an important role in regulating energy homeostasis. Thus far, a number of single nucleotide polymorphisms (SNP) of clock genes have been associated with metabolic disturbances, obesity and dietary habits (e.g., breakfast skipping and macronutrient intakes). Our aim was to examine chronotype associations with SNPs of the 20 known key clock genes which could help to clarify the underlying mechanisms behind chronotype phenotype.
\end{abstract}

Methods: Our data included 8558 participants (aged 25-74 years, 52\% of women) from the cross-sectional population-based National FINRISK 2007 and 2012 surveys. Chronotype was assessed with a shortened six-item version of Horne and Östberg's Morningness-Eveningness Questionnaire (sMEQ) accounting for 83\% of the total variance of the original questionnaire. For comparison we used a single self-evaluation question on chronotype from the questionnaire. Genotyping was done using Illumina arrays (HumanCoreExome, Omniexpress and 610K). Linear and logistic regression was used for statistical analysis with the additive genetic model. The analyses were adjusted for age, sex, batch effects and five principal components to account for population structure. The false discovery rate method by the Benjamini-Yekutieli procedure was applied to correct for multiple comparisons.

Results: We found 27 SNPs in two clock genes that were significantly $(P<0.05)$ associated with chronotype when assessed with the single question. Minor allele carriers of the NR1D2 (Rev-erb $\beta$ ) polymorphisms (rs4131403A, rs4858095T, rs6794922G, rs4619734A, rs4589882A, rs4321479C, rs6779476A, rs6790557A, rs13095392A, rs4858098G, rs4858567C, rs11717862G, rs7431301A, rs5023610G, rs5847265C, rs5847266G, rs6550823A, rs9882735C, rs7371944A, rs55792500G) were associated with evening type, whereas minor allele carriers of the NFIL3 polymorphisms (rs2482704T, rs2989836T, rs2482356C, rs2440589T, rs9409419G, rs2482702G, rs2482357A) were more likely morning types. Findings were similar when chronotype was assessed with sMEQ but did not reach statistical significance.

Discussion: Our findings indicated novel genetic associations of chronotype with two clock genes that have previously been associated with carbohydrate and lipid metabolism (NR1D2), and with lipid absorption and export in intestinal epithelial cells and hepatic gluconeogenesis (NFIL3). These results expand our knowledge of the genetic basis of chronotype and warrant further studies to replicate these findings.

\section{Conflict of Interest}

There is no conflict of interest 